COMMUNICATIONS IN

ANALYSIS AND GEOMETRY

Volume 11, Number 4, 675-695, 2003

\title{
Evolution of radial graphs in hyperbolic space by their mean curvature
}

\section{PhiLip UNTERBERGER ${ }^{1}$}

We consider the evolution of a surface $\mathbf{F}: M^{n} \mapsto \mathcal{H}^{n+1}$ in hyperbolic space by mean curvature flow. That is, we study the one parameter family $\mathbf{F}_{t}=\mathbf{F}(., t)$ of immersions with corresponding images $M_{t}=\mathbf{F}_{t}\left(M^{n}\right)$ such that

$$
\begin{aligned}
\frac{\partial}{\partial t} \mathbf{F}(p, t) & =\mathbf{H}(p, t), \quad p \in M^{n} \\
\mathbf{F}(p, 0) & =\mathbf{F}_{0}(p)
\end{aligned}
$$

where $\mathbf{H}(p, t)$ is the mean curvature vector of the hypersurface $M_{t}$ at $\mathbf{F}(p, t)$ in hyperbolic space.

Mean curvature flow was first studied by Brakke [Bra78] in the context of geometric measure theory. Later, smooth compact surfaces evolving in Euclidean space were investigated in [Hui84] and [Hui90], and on arbitrary ambient manifolds in [Hui86]. The study of the evolution of complete graphs in $\mathcal{R}^{n+1}$ was also studied in [EH89], the results being improved in [EH91].

Minimal and constant mean curvature surfaces in hyperbolic space have also been studied by many authors with Anderson [And82] (see also [And83]) first showing that given any immersed closed submanifold $M^{n-1}$ in the boundary at infinity there exists a complete area minimising current with $M^{n-1}$ as its asymptotic boundary. Hardt and Lin [HL87] then considered the boundary regularity at infinity of these currents after which Lin [Lin89] showed that the regularity of a particular type of minimal graph is the same as that of its asymptotic boundary. During this period first Do Carmo and Lawson [DCL83] and then Levitt and Rosenberg [LR85] classified, under certain assumptions, constant mean curvature surfaces in hyperbolic space.

We will consider only initial surfaces $\mathbf{F}_{0}\left(M^{n}\right)$ which in the upper half space model of hyperbolic space, $\mathcal{H}^{n+1}$, can be written as entire Euclidean

\footnotetext{
${ }^{1}$ This article is published posthumously by Philip Unterberger who passed away on 8 October 2002. In 1998, Phil completed his PhD in Mathematics at the University of Melbourne. This paper is part of his doctoral thesis. Afterwards Phil held a position as a Senior Analyst with the National Australia Bank. His courage and love of life were truly inspirational. He is sadly missed by his wife, daughter, family and all who knew him.
} 
radial graphs above the hyperplane $S_{+}^{n}=S_{+}^{n}(1)$, the Euclidean upper hemisphere of radius one about the origin. That is $\mathbf{F}_{0}\left(M^{n}\right) \subset \mathcal{R}^{n} \times(0, \infty)$ and $\langle\nu, x\rangle>0$ where $\nu$ is an outward normal to $\mathbf{F}_{0}\left(M^{n}\right)$. If $h$ is the log of the Euclidean radial height of $M_{t}$ above $S_{+}^{n}$ and $H^{\mathcal{H}}$ is the mean curvature of the surface as seen from hyperbolic space, then we will see in section 3 that (1) is equivalent up to tangential diffeomorphisms to

$$
\frac{\partial}{\partial t} h=-x_{n+1}\left\langle\nu, \frac{x}{|x|}\right\rangle^{-1} H^{\mathcal{H}}
$$

The term $\left\langle\nu, \frac{x}{|x|}\right\rangle^{-1}$ accounts for the fact that we are flowing in the normal direction rather than in the direction of $x$. This implies that provided we stay in the class of solutions with finite curvature, the asymptotic boundary of a flowing surface is fixed. The geometrical nature of this problem, that of an evolving radial graph in hyperbolic space, suggests its study by calculations on the hypersurface as seen from hyperbolic space.

Our main result, for $B^{n} \subset \mathcal{R}^{n+1}$ an open ball, is

Theorem 0.1. For $M_{0}=F_{0}\left(B^{n}\right)$ a locally Lipschitz continuous entire radial graph over $S_{+}^{n} \subset \mathcal{H}^{n+1}$ the initial value problem (1) has a smooth solution $M_{t}=F_{t}\left(B^{n}\right)$ for all time $t>0$. Moreover each $M_{t}$ is an entire radial graph over $S_{+}^{n}$.

In particular we do not require any initial bounds on either the radial height (defined to be $\log |x|$ ) or gradient. Thus a horosphere for example could be taken as our initial surface and an initial surface can be found whose asymptotic boundary is any given star shaped manifold. If these boundaries are compact and have non-negative mean curvature and we obtain a limit surface $M_{\infty}$ which is minimal then by a uniqueness result of Lin [Lin89] the surface $M_{\infty}$ is one of the type studied by Lin [Lin89]. If we also include the condition that the geodesic height of our initial surface over $S_{+}^{n}$ is bounded we obtain the following convergence result:

Theorem 0.2. If $M_{0}$ has bounded hyperbolic height over $S_{+}^{n}$ then under mean curvature flow $M_{t}$ converges in $C^{\infty}$ to $S_{+}^{n}$.

\section{Remarks.}

1. From Lin [Lin89] any radial graph with asymptotic boundary $\partial M$ which is a minimal surface, is the unique area minimising surface with asymptotic boundary $\partial M$. This can also be shown using a calibration method [Mor87, pg 72]. 
2. There exists a geodesic graph, a surface which intersects each geodesic perpendicular to $S_{+}^{n}$ once, which is rotationally symmetric about the $x_{n+1}$ axis, and which does not remain a graph under mean curvature flow. In fact using a 'clearing out' lemma it can be shown that a neck of this graph pinches under the flow [Unt98].

3. A curve which is a geodesic graph (in $\mathcal{H}^{2}$ ) remains a graph throughout its flow.

I'd like to thank my supervisor Prof. Klaus Ecker for his enthusiasm, patience and support, without which this work would not have been possible. My thanks also to my fellow students, Miles, Nick, and Denis, for many useful and interesting discussions.

\section{Hyperbolic space.}

We begin this section by defining some notation and noting some of the relevant facts about hyperbolic space and the model of it that we will use:

The $(n+1)$-dimensional upper half space model of hyperbolic space is the space $\mathcal{R}^{n} \times(0, \infty)$ endowed with the metric $g_{i j}(x)=\frac{1}{x_{n+1}^{2}} \delta_{i j}$, and we will denote by $\partial \mathcal{H}^{n+1}=\left(\mathcal{R}^{n} \times\{0\}\right) \cup\{\infty\}$ the boundary of its standard compactification. The Riemannian curvature tensor and Ricci and scalar curvatures are given by

$$
R_{i j k l}^{\mathcal{H}}=\frac{1}{x_{n+1}^{4}}\left(\delta_{j k} \delta_{i l}-\delta_{i k} \delta_{j l}\right), R_{i j}^{\mathcal{H}}=-n \frac{1}{x_{n+1}^{2}} \delta_{i j} \text { and } R^{\mathcal{H}}=-n(n+1) .
$$

Expressions in hyperbolic space will, where necessary, be denoted by the subscript or superscript $\mathcal{H}$. We will identify points in $T_{p} \mathcal{R}^{n+1}$ and $T_{p} \mathcal{H}^{n+1}$ so for $\nu$ a unit vector in $T \mathcal{R}^{n+1}, \nu_{\mathcal{H}}=x_{n+1} \nu$ is a unit vector in $T \mathcal{H}^{n+1}$.

Letting $f_{\lambda}(x)=(1+\lambda) x$ we see $\left\langle f_{\lambda}(x), f_{\lambda}(y)\right\rangle_{\mathcal{H}}=\langle x, y\rangle_{\mathcal{H}}$ which makes $f_{\lambda}$ an isometry for all $\lambda$. Differentiating $f_{\lambda}$ with respect to $\lambda$ implies that the vector field $x$ is a Killing vector field. Integral curves of this vector field will be called radial lines, and we define a radial graph to be any hypersurface which intersects each radial line at most once. For a point $p \in M_{t}$ we define the height and gradient of our graph to be

$$
\begin{gathered}
h\left(\frac{p}{|p|}\right)=\log |x(p)| \quad \text { and } \\
v=\langle\nu, \omega\rangle^{-1}=\left\langle\nu_{\mathcal{H}}, \omega_{\mathcal{H}}\right\rangle_{\mathcal{H}}^{-1}=\left(1+|\nabla h|^{2}\right)^{\frac{1}{2}}
\end{gathered}
$$

respectively where $\nu$ is the normal to $M_{t}$ and $\omega=\frac{x}{|x|}$ (We note that the height function used is the one induced by the Killing vector field $x$.) . By 
using

$$
\nabla_{X}^{\mathcal{H}} Y=\nabla_{X} Y+\langle X, Y\rangle_{\mathcal{H}} e_{n+1}^{\mathcal{H}}-\left\langle X, e_{n+1}^{\mathcal{H}}\right\rangle_{\mathcal{H}} Y-\left\langle Y, e_{n+1}^{\mathcal{H}}\right\rangle_{\mathcal{H}} X
$$

(see [GKM75,pg90]) to obtain

$$
\begin{aligned}
\operatorname{div}_{\mathcal{H}} X & =\left\langle\nabla_{e_{i}^{\mathcal{H}}}^{\mathcal{H}} X, e_{i}^{\mathcal{H}}\right\rangle_{\mathcal{H}} \\
& =\left\langle\nabla_{e_{i}} X+\left\langle X, e_{i}\right\rangle_{\mathcal{H}} e_{n+1}^{\mathcal{H}}-\left\langle e_{i}, e_{n+1}^{\mathcal{H}}\right\rangle_{\mathcal{H}} X-\left\langle X, e_{n+1}^{\mathcal{H}}\right\rangle_{\mathcal{H}} e_{i}, e_{i}\right\rangle \\
& =\operatorname{div} X-\left\langle\left\langle X, e_{n+1}^{\mathcal{H}}\right\rangle_{\mathcal{H}} e_{i}, e_{i}\right\rangle \\
& =\operatorname{div} X-\frac{n+1}{2}\left\langle X, \nabla x_{n+1}^{2}\right\rangle_{\mathcal{H}},
\end{aligned}
$$

and so

$$
H^{\mathcal{H}}=x_{n+1} H^{\mathcal{R}}-n\left\langle\nu_{\mathcal{R}}, e_{n+1}\right\rangle,
$$

we are also able to calculate the mean curvature of many graphs quite simply. Finally we also need that the distance between two points, see [BP92], is given by

$$
d^{\mathcal{H}}(p, q)=\cosh ^{-1}\left(\frac{d^{\mathcal{R}}(p, q)^{2}}{2 p_{n+1} q_{n+1}}+1\right) .
$$

We are now able to consider a number of examples of surfaces in hyperbolic space and their evolution under mean curvature flow in some detail. Apart from being interesting in their own right they can be used as barriers to other flowing surfaces and motivate many of the methods used in the remainder of the paper.

The Hyperplane $\mathbf{M}_{\mathbf{0}}=\{\mathbf{x}|| \mathbf{x} \mid=\mathbf{c}\}$.

These appear as Euclidean hemispheres with centres on $\partial \mathcal{H}^{n+1}$ (in particular $S_{+}^{n}$ ) and are radial graphs of constant height, gradient of 1 , and zero mean curvature. Therefore

$$
M_{t}=\{x|| x \mid=c\} .
$$

They are totally geodesic and we consider our graphs to be graphs over $S_{+}^{n}(1)$.

The Horosphere $\mathbf{M}_{\mathbf{0}}=\left\{\mathbf{x} \mid \mathbf{x}_{\mathbf{n}+\mathbf{1}}=\mathbf{c}\right\}$.

This surface is an entire radial graph of unbounded height and gradient. It has constant curvature $-n$ and evolves by translation in the $e_{n+1}$ direction with

$$
M_{t}=\left\{x \mid x_{n+1}=c e^{n t}\right\} .
$$

The Sphere $\mathbf{M}_{\mathbf{0}}=\left\{\mathbf{x} \mid \mathbf{d}^{\mathcal{H}}(\mathbf{p}, \mathbf{x})=\mathbf{r}\right\}$. 
This sphere of radius $r$ with centre $p$ is not a radial graph. It is a compact surface of constant curvature $n \operatorname{coth} r$ and looks like a Euclidean sphere of radius $x_{n+1}(p) \sinh r$. It contracts by homothety to a point with

$$
M_{t}=\left\{x \mid \cosh d^{\mathcal{H}}(p, x)=e^{-n t} \cosh r\right\} .
$$

If we surround any compact minimal surface in $\mathcal{H}^{n+1}$ by a sphere and then flow both surfaces they will touch at some time. By the compact maximum principle this cannot occur and so there don't exist any compact minimal surfaces in $\mathcal{H}^{n+1}$.

The Hypersphere $\mathbf{M}_{0}=\left\{\mathbf{x} \mid \mathbf{x}_{1}^{2}+\ldots+\mathbf{x}_{\mathbf{n}}^{2}+\left(\mathbf{x}_{\mathbf{n}+\mathbf{1}}-\sinh \mathbf{a}_{\mathbf{0}}\right)^{2}=\cosh ^{2} \mathbf{a}_{\mathbf{0}}\right\}$.

These are level sets of the signed distance $a$ from $S_{+}^{n}(1)$, and look like the intersections of Euclidean spheres with the upper half space. They are geodesic graphs of constant curvature $n \tanh a$, remain hyperspheres under (1) with

$$
M_{t}=\left\{x \mid x_{1}^{2}+\ldots+x_{n}^{2}+\left(x_{n+1}-\sinh a_{t}\right)^{2}=\cosh ^{2} a_{t}\right\}
$$

where $\sinh a_{t}=\sinh a_{0} e^{-n t}$, and collapse to $S_{+}^{n}(1)$ in infinite time. So by (2) and a compact maximum principle any surface which evolves for all time and initially lies between two hyperspheres must converge to $S_{+}^{n}(1)$. We note that $\sinh a=\frac{|x|^{2}-1}{2 x_{n+1}}$ for later reference.

The Cylinder $\mathbf{M}_{0}=\left\{\mathbf{x} \mid \frac{|\mathbf{x}|}{\mathrm{x}_{\mathrm{n}+1}}=\mathbf{c}\right\}$.

This surface is found by rotating a radial line about the $x_{n+1}$-axis. It has distance $r$ to the $x_{n+1}$-axis where $\cosh r=\frac{|x|}{x_{n+1}}$, is not a radial graph, and has constant curvature $(n-1) \operatorname{coth} r+\tanh r$. It collapses to the $x_{n+1}$ axis in finite time with

$$
M_{t}=\left\{x \mid \frac{|x|}{x_{n+1}}=\sqrt{\frac{1}{n}\left(1+\left(n c^{2}-1\right) e^{-2 n t}\right)}\right\}
$$

and will be employed in defining cut-off functions for use in interior estimates. In the existance proof for solutions of (1) we will also be required to consider the evolution of our surfaces restricted to various cylinders.

\section{Heat Equations.}

Using methods similar to those in [EH91] we establish interior estimates for the height and all its derivatives. In determining an interior gradient estimate however, we are required to consider a combination of gradient and height which leads to an estimate which increases in time. In order to 
understand how the geometry of $M_{t}$ changes under (1) it will be necessary to calculate $\left(\frac{\partial}{\partial t}-\Delta_{M}\right)$ of many geometric quantities where $\Delta_{M}$ is the LaplaceBeltrami operator on $M_{t}$.

Proposition 2.1. Any function $f: M_{t} \mapsto \mathcal{R}$ on $M_{t} \subset \mathcal{H}^{n+1}$ satisfies

$$
\begin{aligned}
\left(\frac{\partial}{\partial t}-\Delta_{M}\right) f= & -x_{n+1}^{2}\left(\Delta f-\left\langle\nabla_{\nu} \nabla f, \nu\right\rangle\right) \\
& +x_{n+1}\left((n-2)\left\langle\nabla f, e_{n+1}\right\rangle+2\langle\nabla f, \nu\rangle\left\langle\nu, e_{n+1}\right\rangle\right)
\end{aligned}
$$

Proof. For any function $f$ and vector field $\mathrm{X}$

$$
\text { and } \quad \begin{aligned}
\operatorname{div}_{\mathcal{H}} f X & =f \operatorname{div}_{\mathcal{H}} X+X(f), \\
\text { a } \quad \nabla^{\mathcal{H}} f & =x_{n+1}^{2} \nabla f .
\end{aligned}
$$

Making use of (4) and (5) we then have

$$
\begin{aligned}
\left(\frac{\partial}{\partial t}-\Delta_{M}\right) f= & -\operatorname{div}_{M} \nabla^{\mathcal{H}} f \\
= & -\operatorname{div}_{\mathcal{H}} \nabla^{\mathcal{H}} f+\left\langle\nabla_{\nu}^{\mathcal{H}} \nabla^{\mathcal{H}} f, \nu\right\rangle \\
= & -x_{n+1}^{2} \operatorname{div}_{\mathcal{H}} \nabla f-\nabla f\left(x_{n+1}^{2}\right)+x_{n+1}^{2}\left\langle\nabla_{\nu}^{\mathcal{H}} \nabla f, \nu\right\rangle+\langle\nabla f, \nu\rangle \nu\left(x_{n+1}^{2}\right) \\
= & -x_{n+1}^{2} \Delta f+\frac{n+1}{2}\left\langle\nabla f, \nabla x_{n+1}^{2}\right\rangle-\nabla f\left(x_{n+1}^{2}\right) \\
& +x_{n+1}^{2}\left\langle\nabla_{\nu} \nabla f+\langle\nu, \nabla f\rangle_{\mathcal{H}} e_{n+1}-\left\langle\nabla f, e_{n+1}^{\mathcal{H}}\right\rangle_{\mathcal{H}} \nu\right. \\
& \left.-\left\langle\nu, e_{n+1}\right\rangle_{\mathcal{H}} \nabla f, \nu\right\rangle+\langle\nabla f, \nu\rangle\left\langle\nabla x_{n+1}^{2}, \nu\right\rangle \\
= & -x_{n+1}^{2}\left(\Delta f-\left\langle\nabla_{\nu} \nabla f, \nu\right\rangle\right) \\
& +x_{n+1}\left((n-2)\left\langle\nabla f, e_{n+1}\right\rangle+2\langle\nabla f, \nu\rangle\left\langle\nu, e_{n+1}\right\rangle\right)
\end{aligned}
$$

Using this result we now calculate the heat operator of a number of expressions suggested in part by the examples at the end of the first section.

Lemma 2.2. For a radial graph $M_{t}$ evolving by (1) we have

$$
\begin{gathered}
\left(\frac{\partial}{\partial t}-\Delta_{M}\right)|x|^{2}=-4|x|^{2}\left\langle\omega, e_{n+1}\right\rangle\left(\left\langle\omega, e_{n+1}\right\rangle-\langle\nu, \omega\rangle\left\langle\nu, e_{n+1}\right\rangle\right) \\
\left(\frac{\partial}{\partial t}-\Delta_{M}\right) x_{n+1}=(n-2) x_{n+1}+2 x_{n+1}\left\langle\nu, e_{n+1}\right\rangle^{2} \\
\left(\frac{\partial}{\partial t}-\Delta_{M}\right) \sinh a=-n \sinh a, \text { and } \\
\left(\frac{\partial}{\partial t}-\Delta_{M}\right) \cosh r=\frac{1}{\cosh r}\left(1-\langle\nu, \omega\rangle^{2}\right)-n \cosh r .
\end{gathered}
$$

where $a$ is the signed distance from $S_{+}^{n}(1)$ and $r$ is the distance to the $x_{n+1}$ axis. 
Proof. For the proof of (8) we use proposition 2.1 and note

$$
\begin{aligned}
& \nabla x_{n+1}=e_{n+1}, \\
& \nabla_{\nu} \nabla x_{n+1}=0 \text { and } \\
& \Delta x_{n+1}=0 .
\end{aligned}
$$

To prove (9) we proceed similarly noting

$$
\begin{aligned}
& \nabla|x|^{2}=2 x, \\
& \nabla_{\nu} \nabla|x|^{2}=2 \nu \quad \text { and } \\
& \Delta|x|^{2}=2(n+1) .
\end{aligned}
$$

For (10) we use the identity $\sinh a=\frac{|x|^{2}-1}{2 x_{n+1}}$ and calculate

$$
\begin{aligned}
\nabla \frac{|x|^{2}-1}{2 x_{n+1}} & =\frac{x}{x_{n+1}}-\frac{|x|^{2}-1}{2 x_{n+1}^{2}} e_{n+1}, \\
\nabla_{\nu} \nabla \frac{|x|^{2}-1}{2 x_{n+1}}= & \frac{1}{x_{n+1}} \nu-\frac{1}{x_{n+1}^{2}}\left\langle\nu, e_{n+1}\right\rangle x-\frac{1}{x_{n+1}^{2}}\langle x, \nu\rangle e_{n+1} \\
& \quad+\frac{|x|^{2}-1}{x_{n+1}^{3}}\left\langle e_{n+1}, \nu\right\rangle e_{n+1}, \text { and } \\
\Delta \frac{|x|^{2}-1}{2 x_{n+1}}= & \frac{n-1}{x_{n+1}}+\frac{|x|^{2}-1}{x_{n+1}^{3}} .
\end{aligned}
$$

Identity (11) is found noting that $\cosh r=\frac{|x|}{x_{n+1}}$ (see the cylinder example) and using

$$
\begin{aligned}
\nabla \frac{|x|}{x_{n+1}}= & \frac{x}{x_{n+1}|x|}-\frac{|x|}{x_{n+1}^{2}} e_{n+1}, \\
\nabla_{\nu} \nabla \frac{|x|}{x_{n+1}}= & -\frac{1}{x_{n+1}|x|}\langle\nu, \omega\rangle \omega-\frac{1}{x_{n+1}^{2}}\left\langle\nu, e_{n+1}\right\rangle \omega+\frac{1}{x_{n+1}|x|} \nu \\
& \quad+\frac{2|x|}{x_{n+1}^{3}}\left\langle\nu, e_{n+1}\right\rangle e_{n+1}-\frac{1}{x_{n+1}^{2}}\langle\nu, \omega\rangle e_{n+1} \quad \text { and } \\
\Delta \frac{|x|}{x_{n+1}}= & \frac{n-2}{x_{n+1}|x|}+\frac{2|x|}{x_{n+1}^{3} .}
\end{aligned}
$$

To bound the gradient $\langle\nu, \omega\rangle^{-1}$ we need to consider the heat operator of the inner product of the normal and some vector $\vec{u}$ in the direction of the Killing vector field $x$. As there exist examples where the gradient increases during the flow (see [Unt98]) the heat operator of the gradient is not in itself useful. We start by considering $\vec{u}$ to be the Killing vector field $x$ itself. 
Proposition 2.3. For a radial graph $M_{t}$ evolving by mean curvature flow in hyperbolic space we have

$$
\left(\frac{\partial}{\partial t}-\Delta_{M}\right)\left\langle\nu_{\mathcal{H}}, x\right\rangle_{\mathcal{H}}=\left(|A|^{2}-n\right)\left\langle\nu_{\mathcal{H}}, x\right\rangle_{\mathcal{H}}
$$

Proof. We combine the well known formula

$$
\Delta_{M}\left\langle\nu_{\mathcal{H}}, x\right\rangle_{\mathcal{H}}=\left\langle\nabla^{M} H^{\mathcal{H}}, x\right\rangle_{\mathcal{H}}-\left\langle\nu_{\mathcal{H}}, x\right\rangle_{\mathcal{H}} R_{\nu_{\mathcal{H}} \nu_{\mathcal{H}}}^{\mathcal{H}}-\left\langle\nu_{\mathcal{H}}, x\right\rangle_{\mathcal{H}}|A|^{2}-x(H)
$$

(see [Bar84]) where $x(H)$ is the rate with which the mean curvature changes as the manifold is translated along the vector field $x$, with

$$
\begin{aligned}
\frac{\partial}{\partial t}\left\langle\nu_{\mathcal{H}}, x\right\rangle_{\mathcal{H}} & =-H^{\mathcal{H}} \nu_{\mathcal{H}}\left(\left\langle\nu_{\mathcal{H}}, x\right\rangle_{\mathcal{H}}\right) \\
& =\left\langle\nabla^{M} H^{\mathcal{H}}, x\right\rangle_{\mathcal{H}}+H^{\mathcal{H}}\left\langle\nu_{\mathcal{H}}, \nabla_{\nu_{\mathcal{H}}^{\mathcal{H}}}^{\mathcal{H}} x\right\rangle_{\mathcal{H}}
\end{aligned}
$$

to derive

$$
\left(\frac{\partial}{\partial t}-\Delta_{M}\right)\left\langle\nu_{\mathcal{H}}, x\right\rangle_{\mathcal{H}}=\left\langle\nu_{\mathcal{H}}, x\right\rangle_{\mathcal{H}}\left(|A|^{2}+R_{\nu_{\mathcal{H}} \nu_{\mathcal{H}}}^{\mathcal{H}}\right)+x\left(H^{\mathcal{H}}\right)-H^{\mathcal{H}}\left\langle\nabla_{\nu_{\mathcal{H}}}^{\mathcal{H}} x, \nu_{\mathcal{H}}\right\rangle_{\mathcal{H}} .
$$

Since $R_{\nu_{\mathcal{H}} \nu_{\mathcal{H}}}^{\mathcal{H}}=-n$ by $(3)$ and $x\left(H^{\mathcal{H}}\right)=0$ as $x$ is a Killing vector field, we only need to show the last term is 0 . Using (4) we calculate

$$
\begin{aligned}
\left\langle\nabla_{\nu_{\mathcal{H}}}^{\mathcal{H}} x, \nu_{\mathcal{H}}\right\rangle_{\mathcal{H}}= & \left\langle\nabla_{\nu}^{\mathcal{H}} x, \nu\right\rangle \\
= & \left\langle\nabla_{\nu} x, \nu\right\rangle+\frac{1}{x_{n+1}}\left(\langle\nu, x\rangle\left\langle e_{n+1}, \nu\right\rangle-\left\langle\nu, e_{n+1}\right\rangle\langle x, \nu\rangle\right. \\
& \left.\quad-\left\langle x, e_{n+1}\right\rangle\langle\nu, \nu\rangle\right) \\
= & 0
\end{aligned}
$$

since $\left\langle\nabla_{\nu} x, \nu\right\rangle=\langle\nu, \nu\rangle$.

We now look at the heat operator of $\langle\nu, x\rangle$ which is the product of $\left\langle\nu_{\mathcal{H}}, x\right\rangle_{\mathcal{H}}$ and the inverse of the square root of the conformal factor.

Corollary 2.4. Under the conditions as in the previous proposition

$$
\left(\frac{\partial}{\partial t}-\Delta_{M}\right)\langle\nu, x\rangle=\langle\nu, x\rangle|A|^{2}-2\left\langle\nabla^{M}\langle\nu, x\rangle, x_{n+1} e_{n+1}\right\rangle_{\mathcal{H}}
$$


Proof. Combining (9) and (12) implies,

$$
\begin{gathered}
\left(\frac{\partial}{\partial t}-\Delta_{M}\right)\langle\nu, x\rangle=x_{n+1}\left(\frac{\partial}{\partial t}-\Delta_{M}\right)\left\langle\nu_{\mathcal{H}}, x\right\rangle_{\mathcal{H}}+\left\langle\nu_{\mathcal{H}}, x\right\rangle_{\mathcal{H}}\left(\frac{\partial}{\partial t}-\Delta_{M}\right) x_{n+1} \\
\quad-2\left\langle\nabla^{\mathcal{H}} x_{n+1}, \nabla^{\mathcal{H}}\left\langle\nu_{\mathcal{H}}, x\right\rangle_{\mathcal{H}}\right\rangle_{\mathcal{H}} \\
=\left(|A|^{2}-n\right)\langle\nu, x\rangle+(n-2)\langle\nu, x\rangle+2\langle\nu, x\rangle\left\langle\nu, e_{n+1}\right\rangle^{2} \\
\quad-2\left\langle\nabla^{M}\langle\nu, x\rangle-\left\langle\nu_{\mathcal{H}}, x\right\rangle_{\mathcal{H}} \nabla^{M} x_{n+1}, \frac{1}{x_{n+1}} \nabla^{\mathcal{H}} x_{n+1}\right\rangle_{\mathcal{H}} \\
=\langle\nu, x\rangle\left(|A|^{2}-2+2\left\langle\nu, e_{n+1}\right\rangle^{2}\right)-2\left\langle\nabla^{M}\langle\nu, x\rangle, x_{n+1} e_{n+1}\right\rangle_{\mathcal{H}} \\
\quad+2\langle\nu, x\rangle\left(1-\left\langle\nu, e_{n+1}\right\rangle^{2}\right) \\
=\langle\nu, x\rangle|A|^{2}-2\left\langle\nabla^{M}\langle\nu, x\rangle, x_{n+1} e_{n+1}\right\rangle_{\mathcal{H}} .
\end{gathered}
$$

We can now use these results to obtain interior estimates.

Lemma 2.5. Let $M_{t} \subset \mathcal{H}^{n+1}$ be a radial graph evolving by mean curvature flow and $\eta=\cosh R-e^{n t} \cosh r$ where $r$ is the distance from the $x_{n+1}$ axis. Then

$$
\left(\frac{\partial}{\partial t}-\Delta_{M}\right) \eta^{3}\langle\nu, x\rangle^{-1} \leq 2 \eta^{3}\langle\nu, x\rangle^{-1}
$$

on the support of $\eta^{+}$(the positive part of $\eta$ ).

Proof. From (11) we obtain $\left(\frac{\partial}{\partial t}-\Delta_{M}\right) \eta \leq 0$ and $\left(\frac{\partial}{\partial t}-\Delta_{M}\right) \eta^{3} \leq$ $-6 \eta\left|\nabla^{M} \eta\right|^{2}$. Corollary 2.4 then gives

$$
\begin{aligned}
\left(\frac{\partial}{\partial t}-\Delta_{M}\right)\langle\nu, x\rangle^{-1}=- & \langle\nu, x\rangle^{-1}|A|^{2}-2\left\langle\nabla^{M}\langle\nu, x\rangle^{-1}, e_{n+1}^{\mathcal{H}}\right\rangle_{\mathcal{H}} \\
& -2\langle\nu, x\rangle^{-3}\left|\nabla^{M}\langle\nu, x\rangle\right|^{2}
\end{aligned}
$$

Thus

$$
\begin{aligned}
\left(\frac{\partial}{\partial t}-\Delta_{M}\right) \eta^{3}\langle\nu, x\rangle^{-1} \leq- & \eta^{3}\langle\nu, x\rangle^{-1}|A|^{2}-2 \eta^{3}\left\langle\nabla^{M}\langle\nu, x\rangle^{-1}, e_{n+1}^{\mathcal{H}}\right\rangle_{\mathcal{H}} \\
& -2 \eta^{3}\langle\nu, x\rangle^{-3}\left|\nabla^{M}\langle\nu, x\rangle\right|^{2}-6 \eta\langle\nu, x\rangle^{-1}\left|\nabla^{M} \eta\right|^{2} \\
& +6 \eta^{2}\langle\nu, x\rangle^{-2}\left\langle\nabla^{M} \eta, \nabla^{M}\langle\nu, x\rangle\right\rangle_{\mathcal{H}} .
\end{aligned}
$$

The result follows by making the substitutions

$$
-2 \eta^{3}\left\langle\nabla^{M}\langle\nu, x\rangle^{-1}, e_{n+1}^{\mathcal{H}}\right\rangle_{\mathcal{H}} \leq \frac{1}{2} \eta^{3}\langle\nu, x\rangle^{-3}\left|\nabla^{M}\langle\nu, x\rangle\right|^{2}+2 \eta^{3}\langle\nu, x\rangle^{-1}
$$

and

$$
6 \eta^{2}\langle\nu, x\rangle^{-2}\left\langle\nabla^{M} \eta, \nabla^{M}\langle\nu, x\rangle\right\rangle_{\mathcal{H}} \leq 6 \eta\langle\nu, x\rangle^{-1}\left|\nabla^{M} \eta\right|^{2}+\frac{3}{2} \eta^{3}\langle\nu, x\rangle^{-3}\left|\nabla^{M}\langle\nu, x\rangle\right|^{2} .
$$


Lemma 2.6. Let $\omega=\frac{x}{|x|}$ and $R>0$ be such that $\left\{x \in M_{t} \mid e^{n t} \cosh r \leq\right.$ $\cosh R\}$ is a compact radial graph for all $t \in[0, T]$. Then for any $t \in[0, T]$ and $0 \leq \theta<1$ we have the estimate

$\sup _{\left\{x \in M_{t} \mid e^{n t} \cosh r \leq \theta \cosh R\right\}}\langle\nu, \omega\rangle^{-1} \leq e^{2 T+h_{\max }-h_{\min }}(1-\theta)^{-3} \sup _{\left\{x \in M_{0} \mid r \leq R\right\}}\langle\nu, \omega\rangle^{-1}$

where $h_{\max }$ and $h_{\min }$ are the supremum and infimum respectively of the radial height in $\left\{x \in M_{t} \mid r \leq R\right\}$.

Proof. The previous Lemma with ([Ham89]) imply for almost every $t \in$ $(0, T)$

$$
\frac{\partial}{\partial t} \max _{M_{t}} \eta^{3}\langle\nu, x\rangle^{-1} \leq 2 \max _{M_{t}} \eta^{3}\langle\nu, x\rangle^{-1}
$$

Integrating from 0 to $T$ then gives

$$
\max _{M_{T}} \eta^{3}\langle\nu, x\rangle^{-1} \leq e^{2 T} \max _{M_{0}} \eta^{3}\langle\nu, x\rangle^{-1}
$$

and using $h_{\min } \leq \log |x| \leq h_{\max }$ we have

$$
\max _{M_{T}} \eta^{3}\langle\nu, \omega\rangle^{-1} \leq e^{2 T+h_{\max }-h_{\min }} \max _{M_{0}} \eta^{3}\langle\nu, \omega\rangle^{-1}
$$

Since in $\left\{x \in M_{t} \mid e^{n t} \cosh r \leq \theta \cosh R\right\}, \eta^{3} \geq(1-\theta)^{3} \cosh ^{3} R$, we finally obtain by letting $T$ be any $t \in[0, T]$

$\sup _{\left\{x \in M_{t} \mid e^{n t} \cosh r \leq \theta \cosh R\right\}}\langle\nu, \omega\rangle^{-1} \leq e^{2 T+h_{\max }-h_{\min }}(1-\theta)^{-3} \sup _{\left\{x \in M_{0} \mid r \leq R\right\}}\langle\nu, \omega\rangle^{-1}$

Proposition 2.7. For $M_{t} \subset \mathcal{H}^{n+1}$ evolving by mean curvature flow

$$
\begin{aligned}
i \quad\left(\frac{\partial}{\partial t}-\Delta_{M}\right)|A|^{2} & =-2|\nabla A|^{2}+2|A|^{2}\left(|A|^{2}+n\right)-4 H^{2}, \\
i i\left(\frac{\partial}{\partial t}-\Delta_{M}\right)\left|\nabla^{M m} A\right|^{2} & \leq-2\left|\nabla^{M m+1} A\right|^{2} \\
& +C(n, m)\left(\sum_{i+j+k=m}\left|\nabla^{M i} A\right|\left|\nabla^{M j} A\right|\left|\nabla^{M k} A\right|\left|\nabla^{M m} A\right|\right. \\
& \left.+\left|\nabla^{M m} A\right|^{2}\right) .
\end{aligned}
$$

Proof. The first formula was derived in [Hui86] for general manifolds. Direct substitution gives the result in the case of $\mathcal{H}^{n+1}$. From this the second is derived as in [Hui84] and [Ham82, Ch13]. 
Lemma 2.8. Let $M_{t} \subset \mathcal{H}^{n+1}$ evolve by (1) and define

$$
c_{T}=\frac{1}{2 \sup _{[0, T)} \sup _{M_{t}}\langle\nu, x\rangle^{-2}}, \quad \text { and } \quad \varphi=\varphi\left(\langle\nu, x\rangle^{-2}\right)=\frac{\langle\nu, x\rangle^{-2}}{1-c_{T}\langle\nu, x\rangle^{-2}} .
$$

Then

$$
\begin{gathered}
\left(\frac{\partial}{\partial t}-\Delta_{M}\right)|A|^{2} \varphi \leq-2 c_{T}|A|^{4} \varphi^{2}+\left(\frac{c(n)}{c_{T}}-c_{T} \varphi^{\prime}\left|\nabla^{M}\langle\nu, x\rangle^{-1}\right|^{2}\right)|A|^{2} \varphi \\
-\varphi^{-1}\left\langle\nabla^{M} \varphi, \nabla^{M}\left(|A|^{2} \varphi\right)\right\rangle_{\mathcal{H}} .
\end{gathered}
$$

Proof. From Corollary 2.4 we derive

$$
\begin{gathered}
\left(\frac{\partial}{\partial t}-\Delta_{M}\right)\langle\nu, x\rangle^{-2}=-2\langle\nu, x\rangle^{-2}|A|^{2}+4\langle\nu, x\rangle^{-3}\left\langle\nabla^{M}\langle\nu, x\rangle, e_{n+1}^{\mathcal{H}}\right\rangle_{\mathcal{H}} \\
-6\langle\nu, x\rangle^{-4}\left|\nabla^{M}\langle\nu, x\rangle\right|^{2}
\end{gathered}
$$

Following the steps in [EH91, Theorem 3.1] yields the result.

Lemma 2.9. Let $R>0$ be such that $\left\{x \in M_{t} \mid r \leq R\right\}$ is a compact radial graph for any $t \in[0, T]$. Then for any $t \in[0, T]$ and $0 \leq \theta<1$ we have the estimate

$$
\sup _{\left\{x \in M_{t} \mid \cosh r \leq \theta \cosh R\right\}}|A|^{2} \leq c(n)(1-\theta)^{-2}\left(1+\frac{1}{t}\right) \sup _{\left\{x \in M_{s} \mid r \leq R, s \in[0, t]\right\}} v^{4} .
$$

Proof. In this proof we restrict ourselves to the region $\{x \mid r<R\}$ and utilize the methods of [EH91]. Replacing $|A|^{2} \varphi$ by $g$ in the previous lemma leads to

$$
\begin{gathered}
\left(\frac{\partial}{\partial t}-\Delta_{M}\right) g=-2 c_{T} g^{2}+\left(\frac{c(n)}{c_{T}}-c_{T} \varphi^{\prime}\left|\nabla^{M}\langle\nu, x\rangle^{-2}\right|^{2}\right) g \\
-2 \varphi\langle\nu, x\rangle^{3}\left\langle\nabla^{M}\langle\nu, x\rangle^{-1}, \nabla^{M} g\right\rangle_{\mathcal{H}}
\end{gathered}
$$

and for $\eta=(\cosh R-\cosh r)^{2}$ we have using (11)

$$
\left(\frac{\partial}{\partial t}-\Delta_{M}\right) \eta \leq 2 n \cosh ^{2} R-2\left|\nabla^{M} \cosh r\right|^{2}
$$


and $\left|\nabla^{M} \cosh r\right| \leq \sinh r$. Now

$$
\begin{aligned}
-2 \varphi\langle\nu, x\rangle^{3}\left\langle\nabla^{M}\langle\nu, x\rangle^{-1}, \nabla^{M} g\right\rangle_{\mathcal{H}} \eta \leq & 2 \varphi\langle\nu, x\rangle^{3}\left\langle\nabla^{M}\langle\nu, x\rangle^{-1}, \nabla^{M} g \eta\right\rangle_{\mathcal{H}} \\
& +c_{T} \varphi^{\prime}\left|\nabla^{M}\langle\nu, x\rangle^{-1}\right|^{2} g \eta \\
& +\frac{\varphi^{2}}{c_{T} \varphi^{\prime}}\langle\nu, x\rangle^{6} g \frac{\left(\eta^{\prime}\right)^{2}}{\eta}\left|\nabla^{M} \cosh r\right|^{2} \\
= & 2 \varphi\langle\nu, x\rangle^{3}\left\langle\nabla^{M}\langle\nu, x\rangle^{-1}, \nabla^{M} g \eta\right\rangle_{\mathcal{H}} \\
& +c_{T} \varphi^{\prime}\left|\nabla^{M}\langle\nu, x\rangle^{-1}\right|^{2} g \eta \\
& +\frac{4}{c_{T}}\langle\nu, x\rangle^{2} g\left|\nabla^{M} \cosh r\right|^{2} .
\end{aligned}
$$

Thus

$$
\begin{aligned}
\left(\frac{\partial}{\partial t}-\Delta_{M}\right) g \eta=- & 2 c_{T} g^{2} \eta+\left(\frac{c(n)}{c_{T}}-c_{T} \varphi^{\prime}\left|\nabla^{M}\langle\nu, x\rangle^{-2}\right|^{2}\right) g \eta \\
& -2 \varphi\langle\nu, x\rangle^{3}\left\langle\nabla^{M}\langle\nu, x\rangle^{-1}, \nabla^{M} g\right\rangle_{\mathcal{H}} \eta \\
& +2 n g \cosh ^{2} R+6\left|\nabla^{M} \cosh r\right|^{2} g \\
& -2 \eta^{-1}\left\langle\nabla^{M}(g \eta), \nabla^{\mathcal{H}} \eta\right\rangle_{\mathcal{H}} \\
\leq \quad- & 2 c_{T} g^{2} \eta+\frac{c(n)}{c_{T}} g \eta \\
& -2\left\langle\eta^{-1} \nabla^{M} \eta+\varphi\langle\nu, x\rangle^{3} \nabla^{M}\langle\nu, x\rangle^{-1}, \nabla^{\mathcal{H}} g \eta\right\rangle_{\mathcal{H}} \\
& +2 n g \cosh ^{2} R+\left(6+\frac{4}{c_{T}}\langle\nu, x\rangle^{2}\right) 6\left|\nabla^{M} \cosh r\right|^{2} g \\
\leq \quad & 2 c_{T} g^{2} \eta+\frac{c(n)}{c_{T}} g \eta \\
& -2\left\langle\eta^{-1} \nabla^{M} \eta+\varphi\langle\nu, x\rangle^{3} \nabla^{M}\langle\nu, x\rangle^{-1}, \nabla^{\mathcal{H}} g \eta\right\rangle_{\mathcal{H}} \\
& +c(n)\left(1+\frac{\langle\nu, x\rangle^{2}}{c_{T}}\right) g \cosh ^{2} R,
\end{aligned}
$$

Therefore

$$
\begin{aligned}
\left(\frac{\partial}{\partial t}-\Delta_{M}\right) g \eta t \leq- & 2 c_{T} g^{2} \eta t+\left(\frac{c(n)}{c_{T}} t+1\right) g \eta \\
& -2\left\langle\eta^{-1} \nabla^{M} \eta+\varphi\langle\nu, x\rangle^{3} \nabla^{M}\langle\nu, x\rangle^{-1}, \nabla^{\mathcal{H}} g \eta t\right\rangle_{\mathcal{H}} \\
& +c(n)\left(1+\frac{\langle\nu, x\rangle^{2}}{c_{T}}\right) g t \cosh ^{2} R .
\end{aligned}
$$


At a point where $\sup _{0 \leq t \leq T} \sup _{x \in M_{t} \mid r \leq R} t g \eta \neq 0$ is attained for $t_{0}>0$ we compute

$$
2 c_{T} g^{2} \eta t \leq\left(\frac{c(n)}{c_{T}} t+1\right) g \eta+c(n)\left(1+\frac{\langle\nu, x\rangle^{2}}{c_{T}}\right) \cosh ^{2} R g t .
$$

Dividing by $2 c_{T} g$ we compute

$$
g \eta T \leq \frac{1}{2 c_{T}}\left(\frac{c(n)}{c_{T}} T+1\right) \eta+\frac{c(n)}{2 c_{T}}\left(1+\frac{1}{c_{T}}\right) \cosh ^{2} R T,
$$

and since $\eta \leq \cosh ^{2} R$ we have

$$
\begin{aligned}
g \eta T & \leq \frac{1}{2 c_{T}}\left(\frac{c(n)}{c_{T}} T+1\right) \cosh ^{2} R+\frac{c(n)}{c_{T}}\left(1+\frac{1}{c_{T}}\right) \cosh ^{2} R T \\
& \leq \frac{c(n)}{c_{T}^{2}}(1+T) \cosh ^{2} R
\end{aligned}
$$

in the set $\left\{x \in M_{t} \mid r \leq R\right\}$.

As $\eta \geq(1-\theta)^{2} \cosh ^{2} R$ in $\left\{x \in M_{t} \mid \cosh r \leq \theta \cosh R\right\}$ we obtain the required result by replacing $T$ by any $t \in[0, T]$.

Lemma 2.10. Let $R>0$ be such that $\left\{x \in M_{t} \mid r \leq R\right\}$ is a compact radial graph for any $t \in[0, T]$. Then for any $t \in[0, T], m \geq 0$, and $0 \leq \theta<1$ we have the estimate

$$
\sup _{\left\{x \in M_{t} \mid \cosh r \leq \theta \cosh R\right\}}\left|\nabla^{M m} A\right|^{2} \leq c_{m}(1-\theta)^{-2}\left(1+\frac{1}{t}\right)^{m+1}
$$

where $c_{m}=c_{m}\left(n, m, \sup _{\left\{x \in M_{s} \mid r \leq R, s \in[0, t]\right\}} v\right)$.

Proof. Throughout this proof we restrict ourselves to the region $\{x \mid r<R\}$. Lemma 2.9 implies that the above holds for $m=0$. Suppose now that for $R>0$ and $\psi(t)=\frac{t}{t+1}$

$$
\sup _{\left\{x \in M_{t} \mid r \leq R, t \in[0, T]\right\}} \psi^{k}\left|\nabla^{M k-1} A\right|^{2} \leq c_{k-1}
$$

has been established for $0 \leq k \leq m$. We then want to estimate $\psi^{m+1}\left|\nabla^{M m} A\right|^{2}$ in $\left\{x \in M_{t} \mid \cosh r \leq \theta \cosh R, t \in[0, T]\right\}$.

Making use of proposition 2.7 we adopt the same method as in [EH91] with 
$f=\psi^{m+1}\left|\nabla^{M m} A\right|^{2}\left(7 c_{m-1}+1+\psi^{m}\left|\nabla^{M m-1} A\right|^{2}\right)$ and $c=\left(n, m, c_{0}, \ldots, c_{m-1}\right)$ to obtain

$$
\left(\frac{\partial}{\partial t}-\Delta_{M}\right) f \leq-\psi^{-1}\left(\delta f^{2}-c\right)
$$

where $\delta$ has the same dependence as $c$. Using $\eta$ as in lemma 2.9 we have

$$
\begin{array}{r}
\left(\frac{\partial}{\partial t}-\Delta_{M}\right) \eta f \leq-\psi^{-1} \delta \eta f^{2}+\psi^{-1} c \eta+2 n f \cosh ^{2} R \\
-2\left|\nabla^{M} \cosh r\right|^{2} f-2\left\langle\nabla^{M} \eta, \nabla^{\mathcal{H}} f\right\rangle_{\mathcal{H}}
\end{array}
$$

and

$$
-2\left\langle\nabla^{M} \eta, \nabla^{\mathcal{H}} f\right\rangle_{\mathcal{H}}=-4 \eta^{-\frac{1}{2}}\left\langle\nabla^{M} \eta^{\frac{1}{2}}, \nabla^{\mathcal{H}}(\eta f)\right\rangle_{\mathcal{H}}+8\left|\nabla^{M} \cosh r\right|^{2} f
$$

which imply

$$
\begin{gathered}
\left(\frac{\partial}{\partial t}-\Delta_{M}\right) \eta f \leq-\psi^{-1} \delta \eta f^{2}+\psi^{-1} c \eta+2 n f \cosh ^{2} R+6\left|\nabla^{M} \cosh r\right|^{2} f \\
-4 \eta^{-\frac{1}{2}}\left\langle\nabla^{M} \eta^{\frac{1}{2}}, \nabla^{\mathcal{H}}(\eta f)\right\rangle_{\mathcal{H}}
\end{gathered}
$$

Since $\psi(0)=0$ we have $\eta f=0$ at time $t=0$. At the point where, for $t_{0}>0$, $m(T)=\sup _{0<t_{0} \leq T} \sup _{\left\{x \in M_{t} \mid \cosh r \leq \cosh R\right\}} \eta f$ is attained we have

$$
\begin{aligned}
\delta \eta f^{2} & \leq c \eta+6 \psi\left|\nabla^{M} \eta^{\frac{1}{2}}\right|^{2} f+2 n \psi f \cosh ^{2} R \\
& \leq c \eta+6\left|\nabla^{M} \eta^{\frac{1}{2}}\right|^{2} f+2 n f \cosh ^{2} R \\
& \leq c \cosh ^{2} R+6 f \cosh ^{2} R+2 n f \cosh ^{2} R .
\end{aligned}
$$

On $\{x \mid \cosh r \leq \theta \cosh R\}$, the inequality $\eta \geq(1-\theta)^{2} \cosh ^{2} R$ holds and so

$$
\begin{aligned}
(1-\theta)^{2} \delta f^{2} & \leq c+(6+2 n) f \\
& \leq c(1-\theta)^{-2}+(1-\theta)^{2} \frac{\delta}{2} f^{2}
\end{aligned}
$$

$$
\text { which implies } \quad f \leq c(1-\theta)^{2} \text {. }
$$

Thus

$$
\begin{aligned}
& \sup _{\left\{x \in M_{t} \mid \cosh r \leq \theta \cosh R, t \in[0, T]\right\}} \psi^{m+1}\left|\nabla^{M m} A\right|^{2} \leq c_{m}(1-\theta)^{-2} \\
& \text { and } \sup _{\left\{x \in M_{t} \mid \cosh r \leq \theta \cosh R, t \in[0, T]\right\}}\left|\nabla^{M m} A\right|^{2} \leq c_{m}(1-\theta)^{-2}\left(1+\frac{1}{t}\right)^{m+1} \text {. }
\end{aligned}
$$


To find solutions of (1) we are first required to restrict the problem to obtaining solutions over a compact subset of $S_{+}^{n}$.

Define $\Omega_{\varepsilon}=\left\{S_{+}^{n} \mid x_{n+1} \geq \varepsilon\right\}$ and $\Sigma_{R}=\left\{\Omega_{\varepsilon} \mid d(x) \leq R\right\}$ where here $d(x)=d i s t^{E}\left(x, \partial \Omega_{\varepsilon}\right)$ is the Euclidean distance as measured on $\Omega_{\varepsilon}$ from $x$ to $\partial \Omega_{\varepsilon}$. We now find an upper barrier for the flow over $\Omega_{\varepsilon}$. That is we show there exists a surface which lies over $\Sigma_{R}$, above the initial surface and has positive mean curvature. This prevents the gradient of the evolving surface over $\Omega_{\varepsilon}$ from increasing on $\partial \Omega_{\varepsilon}$.

Proposition 2.11. Let $h=\log |x| \in C^{2}\left(\Omega_{\varepsilon}\right)$ be the height of the section of the initial surface, $\mathbf{F}_{0}\left(M^{n}\right)$, which lies over $\Omega_{\varepsilon}$. Then

$$
\delta^{+}=h(x)+\frac{1}{c} \log (1+\beta d(x)) .
$$

for appropriate $c(n, h)$ and $\beta(n, h)$ is an upper barrier for mean curvature flow in $\mathcal{H}^{n+1}$ over $\Omega_{\varepsilon}$.

Proof. [Giu84].

\section{Existence of solutions.}

We reformulate the problem to simplify the analysis. Firstly note that the system

$$
\left(\frac{\partial}{\partial t} \mathbf{F}(p, t)\right)^{\perp}=\mathbf{H}(p, t)
$$

is, up to tangential diffeomorphisms, equivalent to (1). Using a coordinate chart in which the first $n$ coordinates $\vec{\theta}(p, t)$ represent a point on $S_{+}^{n}$ and the $(n+1)^{s t}$ coordinate the Euclidean radial height over $S_{+}^{n}$ we have $\mathbf{F}(p, t)=$ $\left(\vec{\theta}(p, t), e^{h(\vec{\theta}(p, t), t)}\right)$ and $\nu_{\mathcal{H}}=\frac{x_{n+1}(-\nabla h, 1)}{\sqrt{1+|\nabla h|^{2}}}$ so that

$$
\left\langle\frac{\partial}{\partial t} \mathbf{F}, \nu_{\mathcal{H}}\right\rangle_{\mathcal{H}}=\frac{1}{x_{n+1} \sqrt{1+|\nabla h|^{2}}} \frac{\partial}{\partial t} h=-H^{\mathcal{H}}
$$

and

$$
\frac{\partial}{\partial t} h=-x_{n+1}\langle\nu, \omega\rangle^{-1} H^{\mathcal{H}} .
$$


Using $H^{\mathcal{H}}=\operatorname{div}_{M} \nu_{\mathcal{H}}$ we then obtain

$$
\begin{aligned}
\frac{\partial}{\partial t} h= & x_{n+1}^{2}\left(\Delta^{s} h-g^{i k} g^{j l} \frac{\nabla_{i}^{s} h \nabla_{j}^{s} h}{1+\left|\nabla^{s} h\right|^{2}} \nabla_{k}^{s} \nabla_{l}^{s} h\right)-n x_{n+1}\left\langle\nabla^{s} h, e_{n+1}\right\rangle \\
& \quad-\frac{x_{n+1}^{2}\left|\nabla^{s} h\right|^{2}}{\left(1+\left|\nabla^{s} h\right|^{2}\right)} \text { on } S_{+}^{n} \\
h(\vec{\theta}, 0)= & h_{0}(\vec{\theta})
\end{aligned}
$$

where $\Delta^{s}, \nabla^{s}$, and $g^{i j}$, are the Laplacian, gradient and metric coefficients on $S_{+}^{n}$ with the metric induced from $\mathcal{R}^{n+1}$. By projecting down onto $B_{1}^{n}(0) \subset$ $\mathcal{R}^{n}$, defining $s(y)=h\left(\left(y, x_{n+1}\right)\right)$ for $y \in B_{1}^{n}(0)$ we arrive at

$$
\begin{aligned}
\frac{\partial}{\partial t} s & =A\left(x, s, D s, D^{2} s\right) \text { on } B_{1}^{n}(0) \\
s(y, 0) & =s_{0}(y)
\end{aligned}
$$

where

$$
\begin{aligned}
A\left(x, s, D s, D^{2} s\right)= & \left(1-|y|^{2}\right) a^{i j} D_{i} D_{j} s \\
& +\left(1-|y|^{2}\right)\left(n+\frac{1}{1+|D s|^{2}-(D s . y)^{2}}\right) D s . y \\
& +\left(1-|y|^{2}\right) \frac{|D s|^{2}-(D s . y)^{2}}{1+|D s|^{2}-(D s . y)^{2}}
\end{aligned}
$$

and

$$
a^{i j}=\delta^{i j}-y^{i} y^{j}-\frac{(D s-(D s . y) y)^{i}(D s-(D s . y) y)^{j}}{1+|D s|^{2}-(D s . y)^{2}} .
$$

The coefficient matrix $a^{i j}$ has :

1. For $y \| D s$ an eigenvalue $\frac{1-|y|^{2}}{1+\left(1-|y|^{2}\right)|D s|^{2}}$ with multiplicity 1 and an eigenvalue 1 with multiplicity $n-1$.

2. For $y \perp D s$ eigenvalues $1-|y|^{2}$ and $\frac{1}{1+|D s|^{2}}$ with multiplicity 1 and an eigenvalue 1 with multiplicity $n-2$, and

3. Otherwise two eigenvalues

$$
\frac{\left(1-|y|^{2}\right)\left(1+|D s|^{2}\right)+1 \pm \sqrt{\left(1-\left(1-|y|^{2}\right)\left(1+|D s|^{2}\right)\right)^{2}+4\left(1-|y|^{2}\right)(D s . y)^{2}}}{2\left(1+|D s|^{2}-(D s . y)^{2}\right)}
$$

with multiplicity 1 and an eigenvalue 1 with multiplicity $n-2$.

Clearly as $|y| \rightarrow 1$ or $|D s| \rightarrow \infty$ some eigenvalues may become zero and thus our equation may not be uniformly parabolic. This is one of the main problems in proving existence of a solution of the flow. 
Theorem 3.1. Suppose $F_{0}\left(M_{\varepsilon}^{n}\right)$ is a smooth radial graph over $\Omega_{\varepsilon}=$ $\left\{S_{+}^{n} \mid x_{n+1} \geq \varepsilon\right\}$. Then the initial value problem

$$
\begin{array}{lc}
\frac{\partial}{\partial t} \mathbf{F}(p, t)=\mathbf{H}(p, t), & p \in M_{\varepsilon}^{n} \\
\mathbf{F}(p, 0)=\mathbf{F}_{0}(p), & p \in M_{\varepsilon}^{n} \\
\mathbf{F}(p, t)=\mathbf{F}_{0}(p), & p \in \partial M_{\varepsilon}^{n}
\end{array}
$$

has a smooth radial graph solution for all time.

Proof. The result follows if we have a smooth solution for all time to the problem

$$
\begin{array}{lrl}
\frac{\partial}{\partial t} s=A\left(x, s, D s, D^{2} s\right), & y & \in B^{n} \\
s(y, 0)=s_{0}(y), & y & \in B^{n} \\
s(y, t)=s_{0}(y), & y & \in \partial B_{\sqrt{1-\varepsilon^{2}}}^{n}(0) .
\end{array}
$$

As $|y| \leq \sqrt{1-\varepsilon^{2}}$, this system is uniformly parabolic (see eigenvalues on previous page) provided $|D s|<\infty$. Given these the results of Ladyzhenskaya et al. [LSU68] apply and yield a smooth solution of (15). Since $|D s|^{2} \leq \frac{|\nabla h|^{2}}{\varepsilon^{2}}=\frac{v^{2}-1}{\varepsilon^{2}}$ our result follows provided $v^{-1}=\langle\nu, \omega\rangle$ is bounded away form zero. Employing Corollary 2.4 and a compact maximum principle shows $\langle\nu, x\rangle$ takes its minimum on the parabolic boundary $B_{\sqrt{1-\varepsilon^{2}}}^{n}(0) \times 0 \cup \partial B_{\sqrt{1-\varepsilon^{2}}}^{n}(0) \times(0, \infty)$. Initial bounds and the barriers (Proposition 2.11) imply $\langle\nu, x\rangle$ is bounded here. The barrier $S_{+}^{n}\left(e^{h_{\max }}\right)$ is used to bound $|x|$ above, and so $\langle\nu, \omega\rangle$ is bounded away from zero completing the proof.

Theorem 3.2. Let $M_{0}=F_{0}\left(B^{n}\right)$ be a locally Lipschitz continuous entire radial graph over $S_{+}^{n} \subset \mathcal{H}^{n+1}$. Then the initial value problem (1) has a smooth solution $M_{t}=F_{t}\left(B^{n}\right)$ for all time $t>0$. Moreover, each $M_{t}$ is an entire graph over $S_{+}^{n}$.

Proof. We first assume $M_{0}$ is smooth. For the solid cylinder $C_{\varepsilon}=$ $\left\{x \mid \frac{|x|}{x_{n+1}} \leq \frac{1}{\varepsilon}\right\}$, let $M_{t}^{\varepsilon}$ be the solution of (14) with $M_{\varepsilon}^{n}=\mathbf{F}_{0}^{-1}\left(M_{0} \cap C_{\varepsilon}\right)$ which by Theorem 3.1 exists for all time, and let $h_{t}^{\varepsilon}$ and $s_{t}^{\varepsilon}$ be the radial height of $M_{t}^{\varepsilon}$ over $S_{+}^{n}$ and its projection onto a ball in $\mathcal{R}^{n}$ respectively.

Denote now by $B_{R}^{n}$ the restriction of the hyperbolic ball of radius $R$ and center $(\overrightarrow{0}, 1)$ to $S_{+}^{n}$. Then choose $R_{0}$ so that $B_{4 R_{0}}^{n} \subset \subset \Omega_{\varepsilon}$. For $h^{m}=$ $\sup _{\left\{x \in M_{0} \mid r<4 R_{0}\right\}} h_{0}^{\varepsilon}$ we find (see "The Sphere" example)

$$
M_{0}^{\varepsilon} \cap B_{4 R_{0}}^{n+1}\left(p^{+}\right)=\emptyset
$$


where $p^{+}=\left(\overrightarrow{0}, \frac{1+e^{h^{m}}}{\cosh 4 R_{0}-\sinh 4 R_{0}}\right)$. Furthermore

$$
\partial M_{t}^{\varepsilon} \cap B_{4 R_{0}}^{n+1}\left(p^{+}\right)=\emptyset .
$$

Using a compact maximum principle (see Lemma 3.2 of [Hui86]) and "The Sphere" example we infer

$$
M_{t}^{\varepsilon} \cap B_{2 R_{0}}^{n+1}\left(p^{+}\right)=\emptyset
$$

for $t \in\left[0, t_{0}\right]$ where $t_{0}=\frac{1}{n} \log \frac{\cosh 4 R_{0}}{\cosh 2 R_{0}}$. Using a similar argument with $B_{4 R_{0}}^{n+1}\left(p^{-}\right)$where $p^{-}=\left(\overrightarrow{0}, \frac{e^{h_{m}}}{2 \cosh R+\sinh R}\right)$ and $h_{m}=\inf _{\left\{x \in M_{0} \mid r<4 R_{0}\right\}} h_{0}^{\varepsilon}$ we conclude that the height function satisfies

$$
\sup _{B_{2 R_{0}}^{n} \times\left[0, t_{0}\right]}\left|s_{t}^{\varepsilon}\right|<c_{0}
$$

where $c_{0}$ depends only on $n, R_{0}$ and $\sup _{B_{4 R_{0}}^{n} \times\{0\}}\left|h_{0}^{\varepsilon}\right|$.

Applying the gradient estimate of Lemma 2.6 we obtain

$$
\sup _{B_{\frac{3}{2} R_{0}}^{n} \times\left[0, t_{1}\right]}\left|D s_{t}^{\varepsilon}\right| \leq c_{1}
$$

where $t_{1}<t_{0}$ and $c_{1}=c_{1}\left(n, R_{0}, c_{02}, \sup _{B_{2 R_{0}}^{n} \times\{0\}}\left|D s_{t}^{\varepsilon}\right|\right)$. We note that as $R_{0} \rightarrow \infty$ both $t_{0}$ and $t_{1}$ may approach $\infty$. From Lemma 2.10 we then conclude for any integer $m \geq 0$

$$
\sup _{B_{R_{0}}^{n} \times\left[0, t_{1}\right]}\left|D^{m} s_{t}^{\varepsilon}\right| \leq c_{m}
$$

where $c_{m}=c_{m}\left(m, n, R_{0}, c_{1}\right)$.

Since $R_{0}$ and $t_{1}$ are arbitrary we can select a sequence of solutions $\left(s_{t}^{\varepsilon_{i}}\right)$ for $\varepsilon_{i} \rightarrow 0\left(\varepsilon_{i}<\varepsilon\right)$ such that $s_{t} \stackrel{i \rightarrow \infty}{\rightarrow} s$ in $C^{\infty}$ uniformly on compact subsets of $M^{n} \times(0, \infty)$. This establishes the existence of a family of entire graphs $M_{t}=$ graph $h_{t}$ solving (1) where $h \in C^{\infty}\left(S_{+}^{n} \times(0, \infty)\right)$. As the second and higher order derivative estimates for $h$ on each compact subset of $S_{+}^{n}$ depend only on the initial gradient on a slightly larger subset, an approximation argument yields a smooth solution of (1) also for locally Lipschitz initial data.

Theorem 3.3. If $M_{0}$ has bounded gradient and hyperbolic height over $S_{+}^{n}$ then under mean curvature flow $M_{t}$ converges in $C^{\infty}$ to $S_{+}^{n}$. 
Proof. As $M_{0}$ has bounded hyperbolic height it lies between two hyperspheres (see "The Hypersphere" example). In view of (2), the solution $M_{t}$ does not move at the boundary and therefore by the compact maximum principle remains between the evolving barriers. Since both barriers evolve to $S_{+}^{n}$ our solution converges uniformly to $S_{+}^{n}$ in height.

Allowing $R \rightarrow \infty$ in lemma 2.6 we infer that $v$ remains bounded on some small time interval $[0, T]$ and hence by letting $R \rightarrow \infty$ in the interior estimate for $|A|^{2}$, lemma 2.9 , that $|A|^{2}$ is bounded on some small time interval $\left[t_{0}, T\right]$ for $t_{0}>0$. We may thus use the non compact maximum principle [EH91, Theorem 4.3] which with the equation for the heat operator of $\langle\nu, x\rangle^{-1}$, Corollary 2.4, imply that $\langle\nu, x\rangle^{-1}$ is bounded above on $\left[t_{0}, T\right]$ by its maximum value at time $t_{0}$. It follows that $\langle\nu, x\rangle^{-1}$ is bounded above by its initial maximum for all time. Our height bounds then imply that $v$ is bounded independent of time. From the interior estimate, lemma 2.10, with $R \rightarrow \infty$ we then obtain bounds for $\left|\nabla^{M m} A\right|^{2}$ for $m \geq 0$ independent of time. Thus height and all its derivatives are bounded independent of time. Employing the Theorem of Arzela-Ascoli the result follows.

\section{References.}

[And82] M. Anderson. Complete minimal varieties in hyperbolic space. Invent.Math., 69 477-494, 1982.

[And83] M. Anderson. Complete minimal hypersurfaces in hyperbolic nmanifolds. Comment. Math. Helv., 58264-290, 1983.

[Bar84] R. Bartnik. Existence of maximal surfaces in asymptotically flat spacetimes. Communications in Mathematical Physics, 94:155$175,1984$.

[BP92] R. Benedetti and C. Petronio. Lectures on Hyperbolic Geometry. SpringerVerlag, 1992

[Bra78] K. A. Brakke. The Motion of a Surface by its Mean Curvature. Princeton University Press, 1978.

[DCL83] M. Do Carmo and H. Lawson, Jr. On Alexandrov-Bernstein theorems in hyperbolic space. Duke Math. J., 50 995-1003, 1983.

[EH89] K. Ecker and G. Huisken. Mean curvature evolution of entire graphs. Ann. Math., 130 453-471, 1989. 
[EH91] K. Ecker and G. Huisken. Interior estimates for hypersurfaces moving by mean curvature. Invent. Math., 105 547-569, 1991.

[Giu84] E. Giusti. Minimal surfaces and functions of bounded variation, volume 80 of Monographs in Mathematics. Birkhauser, 1984.

[GKM75] D. Gromoll, W. Klingenberg, and W. Meyer. Riemannsche Geometrie im Grossen, volume 55 of Lecture Notes in Mathematics. Springer-Verlag, 1975.

[Ham82] R. S. Hamilton. Three-manifolds with positive Ricci curvature. J. Differ.Geom., 17 255-306, 1982.

[Ham89] R. S. Hamilton. The maximum principle. Honolulu, Hawaii (lecturenotes), 1989.

[HL87] R. Hardt and F.H. Lin. Regularity at infinity for area-minimising hypersurfaces in hyperbolic space. Invent. Math., 88 217-224, 1987.

[Hui84] G. Huisken. Flow by mean curvature of convex surfaces into spheres. J.Differ. Geom., 20 237-266, 1984.

[Hui86] G. Huisken. Contracting convex hypersurfaces in Riemannian manifolds by their mean curvature. Invent. Math., 84 463-480, 1986 .

[Hui90] G. Huisken. Asymptotic behaviour for singularities of the mean curvature flow. J. Differ. Geom.,31285-299, 1990.

[Lin89] F.H. Lin. On the Dirichlet problem for minimal graphs in hyperbolic space. Invent Math., 96 593-612, 1989.

[LR85] G. Levitt and H. Rosenberg. Symmetry of constant mean curvature hypersurfaces in hyperbolic space. Duke Math. J.,52 53-59, 1985 .

[LSU68] O. A. Ladyzhenskaya, V. A. Solonnikov, and N. N. Uraltseva. Linear and Quasilinear Equations of parabolic Type Translation, volume 23 of II. Ser. Am. Math. Soc., 1968.

[Mor87] F. Morgan. Geometric measure theory - A beginner's guide. Academic press inc., 1987. 
[Unt98] P. Unterberger. Mean curvature evolution in hyperbolic space. Ph.D. Thesis. University of Melbourne, Australia, 1998.

University of Melbourne

PARKVILle, Victoria 3052

Australia 